\title{
Extensional rewriting with sums
}

\author{
Sam Lindley \\ Laboratory for Foundations of Computer Science, \\ School of Informatics, The University of Edinburgh \\ Sam.Lindley@ed.ac. uk ${ }^{\star}$
}

\begin{abstract}
Inspired by recent work on normalisation by evaluation for sums, we propose a normalising and confluent extensional rewriting theory for the simply-typed $\lambda$-calculus extended with sum types. As a corollary of confluence we obtain decidability for the extensional equational theory of simply-typed $\lambda$-calculus extended with sum types. Unlike previous decidability results, which rely on advanced rewriting techniques or advanced category theory, we only use standard techniques.
\end{abstract}

\section{Introduction}

It is easy to add sum types to the equational theory of the simply-typed $\lambda$ calculus, in the presence of $\eta$-rules, or to add sum types to the rewriting theory of simply-typed $\lambda$-calculus, in the absence of $\eta$-rules. However, adding sum types to the rewriting theory is difficult in the presence of $\eta$-rules. Existing rewriting theories, with the exception of Ghani's [5], are either incomplete with respect to the equational theory or non-confluent. Quoting Altenkirch et al [1], Ghani's work involves 'intricate rewriting techniques whose details are daunting'. Our aim is to introduce a straightforward rewriting theory using standard techniques.

The essential reason why the problem with confluence arises is that reordering independent nested cases does not change the semantics of a term. For instance, let $=$ be equivalence in the equational theory, writing $\delta\left(p, x_{1} \cdot n_{2}, x_{2} \cdot n_{2}\right)$ for case $\mathrm{p}$ of $\mathrm{in}_{1}\left(\mathrm{x}_{1}\right) \Rightarrow \mathrm{n}_{2} \mid \mathrm{in}_{2}\left(\mathrm{x}_{2}\right) \Rightarrow \mathrm{n}_{2}$, then

$$
\begin{aligned}
& \delta\left(p_{1}, x_{1} \cdot \delta\left(p_{2}, y_{1} \cdot n_{1}, y_{2} \cdot n_{2}\right), x_{2} \cdot \delta\left(p_{2}, y_{1} \cdot m_{1}, y_{2} \cdot m_{2}\right)\right) \\
& \quad=\delta\left(p_{2}, y_{1} . \delta\left(p_{1}, x_{1} \cdot n_{1}, x_{2} \cdot m_{1}\right), y_{2} \cdot \delta\left(p_{1}, x_{1} \cdot n_{2}, x_{2} \cdot m_{2}\right)\right)
\end{aligned}
$$

where $x_{1}, x_{2}, y_{1}, y_{2}, m_{1}, m_{2}, n_{1}, n_{2}, p_{1}, p_{2}$ are distinct object variables. The structure of the terms on each side of the equation is identical, so it is not possible to capture the equivalence with a rewrite rule.

This article explores several solutions to the case ordering problem, all suggested by the work of Altenkirch et al [1] and Balat et al [2] on normalisation by evaluation for the simply-typed $\lambda$-calculus extended with sums. The goal of normalisation by evaluation [4] is to find a unique normal form with respect to the equational theory. In contrast, we shall be interested in normal forms with respect to a rewriting theory.

In fact, the case ordering problem also manifests itself in the equational setting. In the example above, either the left hand side or the right hand side

\footnotetext{
* Supported by EPSRC grant number EP/D046769/1
} 
of the equivalence should be a normal form. But the terms are structurally identical so some non-structural property must be used to define normal forms. One possibility is to define an ordering on terms via an ordering on variable names. The ordering on terms can then be used to assign an ordering to nested cases. Such an ordering is undesirable as it requires us to dispense with $\alpha$ conversion.

Altenkirch et al solve the problem by adding a new construct to the object language - a parallel case that simultaneously eliminates a set of sums. Using the extended language both sides of the equivalence are represented by the same parallel case. A big advantage of this approach is that it leads to a syntax which much more closely captures the semantics of the calculus. The main disadvantage is that it drastically increases the complexity of the machinery used by the syntax of the language. In Altenkirch et al's presentation functions appear in the syntax. These functions can be represented more concretely using sets or lists, but the resulting syntax is still significantly more complex than the standard one.

Balat et al 2] build on Altenkirch et al's work. Instead of adding parallel cases, they define a congruence over terms which contains the equivalence given in the example above as a special case. They identify normal forms up to this congruence, leading to a rather elegant presentation.

We adopt an extension $\sim$ of Balat et al's congruence, and perform rewriting modulo $\sim$. Sect. 2 introduces the equational theory $\lambda^{\rightarrow \times+}$ of simply-typed lambda-calculus extended with products and sums, and decomposes the general $\eta$ axiom for sums into a number of simpler axioms. Sect. 3 describes a non-local rewriting theory that generates the equational theory. Sect. 4 gives a reducibility proof of strong normalisation for a fragment of the rewriting theory following the approach of Lindley and Stark [8, Chaper 3] 9]. Sect. 5 uses strong normalisation results for fragments of the rewriting theory to prove weak normalisation and confluence modulo $\sim$ for the full rewriting theory, and hence decidability for the equational theory. Sect. 6 describes three variations of the rewriting theory. Sect. 7 concludes.

\section{The object language}

The simply typed lambda calculus extended with products and sums is standard [5]. We write $\lambda \rightarrow \times+$ for the equational theory.

$$
\text { (Types) } \quad A, B::=\mathrm{O}|\mathrm{A} \rightarrow \mathrm{B}| \mathrm{A} \times \mathrm{B} \mid \mathrm{A}+\mathrm{B}
$$

Types are constructed from a base type $\mathrm{O}$, functions $\mathrm{A} \rightarrow \mathrm{B}$ from type $\mathrm{A}$ to type $B$, products $A \times B$ of types $A$ and $B$, and sums of types $A$ and $B$. We omit the unit and empty types, but restoring them does not radically change our proofs (though the empty type requires a little more care in the handling of typing 
contexts).

(Terms)

$$
\begin{aligned}
\mathrm{m}, \mathrm{n}, \mathrm{p}::=x \\
\\
|\lambda x . \mathrm{m}| \mathrm{mn}|\langle\mathrm{m}, \mathfrak{n}\rangle| \pi_{1}(\mathrm{~m}) \mid \pi_{2}(\mathrm{~m}) \\
\left|\mathfrak{l}_{1}(\mathrm{~m})\right| \mathfrak{l}_{2}(\mathrm{~m}) \mid \delta\left(\mathrm{m}, x_{1} \cdot \mathrm{n}_{1}, \mathrm{x}_{2} . \mathrm{n}_{2}\right)
\end{aligned}
$$

Terms are constructed from variables $x$, lambda abstractions $\lambda x . m$, applications $m n$, pairs $\langle m, n\rangle$, projections $\pi_{i}(m)$, injections $\mathfrak{l}_{i}(m)$ and cases $\delta\left(m, x_{1} \cdot n_{1}, x_{2} . n_{2}\right)$. Terms are identified up to $\alpha$-conversion.

The free $f v(m)$ and bound variables $b v(m)$ are defined in the usual way. We write $m[x:=n]$ for the capture-avoiding substition of $n$ for $x$ in $m$, and $\mathfrak{m}\left[\mathrm{x}_{1}:=\mathrm{n}_{1}, \ldots, \mathrm{x}_{\mathrm{k}}:=\mathrm{n}_{\mathrm{k}}\right]$ for the simultaneous capture-avoiding substition of $n_{i}$ for $x_{i}$ in $m(1 \leqslant i \leqslant k)$. We write size $(m)$ for the size of the term $m$. The typing rules are standard. Each type constructor has an introduction rule and an elimination rule.

$$
\begin{gathered}
\overline{\Gamma, x: A \vdash x: A} \quad \frac{\Gamma, x: A \vdash m: B}{\Gamma \vdash \lambda x \cdot m: A \rightarrow B} \quad \frac{\Gamma \vdash m: A \rightarrow B \quad \Gamma \vdash n: A}{\Gamma \vdash m n: B} \\
\frac{\Gamma \vdash m: A \quad \Gamma \vdash n: B}{\Gamma \vdash\langle m, n\rangle: A \times B} \quad \frac{\Gamma \vdash m: A_{1} \times A_{2}}{\Gamma \vdash \pi_{i}(m): A_{i}} \quad i \in\{1,2\} \\
\frac{\Gamma \vdash m: A_{i}}{\Gamma \vdash \mathfrak{l}_{i}(m): A_{1}+A_{2}} \quad i \in\{1,2\} \\
\frac{\Gamma \vdash m: A_{1}+A_{2} \quad \Gamma, x_{i}: A_{i} \vdash n_{i}: B \quad i \in\{1,2\}}{\Gamma \vdash \delta\left(m, x_{1} \cdot n_{1}, x_{2} \cdot n_{2}\right): B}
\end{gathered}
$$

Axioms The axioms for $\lambda^{\circ+}$ consist of a $\beta$-axiom and an $\eta$-axiom for each type constructor.

$$
\begin{array}{ll}
(\rightarrow . \beta) & (\lambda x . m) n=m[x:=n] \\
\left(\times . \beta_{i}\right) & \pi_{i}\left(\left\langle m_{1}, m_{2}\right\rangle\right)=m_{i}, \quad i \in\{1,2\} \\
\left(+. \beta_{i}\right) & \delta\left(\iota_{i}(m), x_{1} \cdot n_{1}, x_{2} \cdot n_{2}\right)=n_{i}\left[x_{i}:=m\right], \quad i \in\{1,2\} \\
(\rightarrow . \eta) & m=\lambda x \cdot m x, \quad x \notin f v(m) \\
(\times . \eta) & m=\left\langle\pi_{1}(m), \pi_{2}(m)\right\rangle \\
\left(+. \eta^{\dagger}\right) & n[x:=p]=\delta\left(p, x_{1} \cdot n\left[x:=\iota_{1}\left(x_{1}\right)\right], x_{2} \cdot n\left[x:=\iota_{2}\left(x_{2}\right)\right]\right)
\end{array}
$$

The equation $m=n$ is shorthand for the equality judgement $\Gamma \vdash m=n: A$ where $\Gamma \vdash \mathrm{m}: A$ and $\Gamma \vdash \mathrm{n}: A$. The equational theory is given by the least (typed) congruence satisfying the axioms.

Alternative axioms The generalised $\eta$-axiom for sums.$+ \eta^{\dagger}$ is non-local and it is not at all obvious how it might give rise to a confluent rewriting system. In particular, note that substitutions appear both on the left and the right hand 
side of the axiom. We break.$+ \eta^{\dagger}$ down into a number of simpler axioms.

$$
\begin{aligned}
& (+. \eta) \quad p=\delta\left(p, x_{1} \cdot \iota_{1}\left(x_{1}\right), x_{2} \cdot \iota_{2}\left(x_{2}\right)\right) \\
& (\text { move-case }) \quad F\left[\delta\left(p, x_{1} \cdot n_{1}, x_{2} \cdot n_{2}\right)\right]=\delta\left(p, x_{1} \cdot F\left[n_{1}\right], x_{2} \cdot F\left[n_{2}\right]\right), \\
& \quad x_{1}, x_{2} \notin f v(F[]) \text { and } b v(F[]) \cap f v(p)=\emptyset \\
& (\text { repeated-guard) } \\
& \quad \delta\left(p, x_{1} \cdot \delta\left(p, y_{1} \cdot n_{1}, y_{2} \cdot n_{2}\right), x_{2} \cdot \delta\left(p, z_{1} \cdot p_{1}, z_{2} \cdot p_{2}\right)\right) \\
& \quad=\delta\left(p, x_{1} \cdot n_{1}\left[y_{1}:=x_{1}\right], x_{2} \cdot p_{2}\left[z_{2}:=x_{2}\right]\right), \quad x_{1}, x_{2} \notin f v(p) \\
& \left(\text { redundant-guard) } \quad \delta\left(p, x_{1} \cdot n, x_{2} \cdot n\right)=n, \quad x_{1}, x_{2} \notin f v(n)\right.
\end{aligned}
$$

The local $\eta$ axiom for sums.$+ \eta$ is a special case of.$+ \eta^{\dagger}$ in which $n$ is just $x$. The move-case axiom is a generalisation of the usual commuting conversions for $\lambda \rightarrow \times+6\left[11\right.$. As well as allowing cases to move across elimination frames $\left(\mathrm{F}_{1}[\mathrm{]})\right.$, move-case also allows them to be moved across neutral frames $\left(\mathrm{F}_{2}[]\right)$, lambda frames $\left(\mathrm{F}_{3}[]\right)$ and continuation frames $\left(\mathrm{F}_{4}[]\right)$.

$$
\begin{array}{ll}
\text { (Frames) } & F[]::=F_{1}[]\left|F_{2}[]\right| F_{3}[] \mid F_{4}[] \\
& F_{1}[]::=[] n\left|\pi_{1}([])\right| \pi_{2}([]) \mid \delta\left([], x_{1} \cdot n_{1}, x_{2} \cdot n_{2}\right) \\
& F_{2}[]::=m[]|\langle[], n\rangle|\langle m,[]\rangle\left|\iota_{1}([])\right| \iota_{2}([]) \\
& F_{3}[]::=\lambda x \cdot[] \\
& F_{4}[]::=\delta\left(p, x_{1} \cdot[], x_{2} \cdot n_{2}\right) \mid \delta\left(p, x_{1} \cdot n_{1}, x_{2} \cdot[]\right)
\end{array}
$$

We write move-case $i$ for the restriction of move-case to frames of the form $F_{i}$. Following Altenkirch et al, we use the word guard to refer to the first argument of a case. The axiom repeated-guard allows guards to be copied or deleted. The axiom redundant-guard is a special case of.$+ \eta^{\dagger}$ in which $x$ does not occur free in $n$.

Proposition 1. Replacing the axiom.$+ \eta^{\dagger}$ with the alternative axioms.$+ \eta$, move-case, repeated-guard, redundant-guard yields the same equational theory.

Proof. (sketch)

New axioms are sound:

.$-+ \eta$ and redundant-guard are instances of.$+ \eta^{\dagger}$

- move-case, repeated-guard: apply.$+ \eta^{\dagger}$ from left to right using $p$ as the substituted term, eliminate resulting.$+ \beta_{i}$ redexes, then $\alpha$-convert

New axioms are complete:

- $\eta$ expand all instances of $p$ in $n$

- use move-case to hoist all instances of $p$ to the top

- use repeated-guard and redundant-guard to get rid of the multiple copies of $\mathrm{p}$

- use redundant-guard for the case where $x \notin f v(n)$ 
The axioms move-case, repeated-guard and redundant-guard, are implicit in previous work on normalisation by evaluation with sums [12]. To the author's knowledge, they have not previously been used as the basis for a rewriting calculus.

\section{A rewriting theory}

As a first attempt at a rewriting theory consider defining a rewrite rule for each axiom by orienting from left to right (with the usual restrictions for $\eta$-expansion). Unfortunately the resulting theory has infinite reduction sequences arising from move-case ${ }_{4}$. For instance, the following reductions can be applied indefinitely as $m$ appears as a subterm of $n$.

$$
\begin{aligned}
& m=\delta(p, x . n, x . \delta(p, x . n, x . n)) \\
& \longrightarrow \text { move-case }_{4} \delta(p, x . \delta(p, x . n, x . n), x . \delta(p, x . n, x . n)) \\
& \longrightarrow \text { move-case }_{4} \delta(p, x . m, x . m)=n
\end{aligned}
$$

Ohta and Hasegawa 10 face a similar problem for a linear lambda calculus. Their solution is to separate the axioms of their equational theory into a reduction relation and an equivalence relation, and use Huet's technique for proving confluence of the reduction relation modulo the equivalence relation 7 . Balat et al use an equivalence for defining normal forms and implementing normalisation by evaluation with sums. Their equivalence is the least congruence satisfying the move-case $_{4}$ and redundant-guard axioms. We introduce a congruence that also includes the repeated-guard axiom.

Definition 2. The relation $\sim$ is the least congruence satisfying the axioms move-case ${ }_{4}$, repeated-guard and redundant-guard.

Deciding equivalence modulo $\sim$ is straightforward. First we define some auxiliary functions.

\section{Definition 3.}

$$
\begin{aligned}
& \text { Guards }(\mathrm{m})= \\
& \begin{cases}p \cup \operatorname{Guards}\left(\mathrm{x}_{1} \cdot \mathrm{n}_{1}\right) \cup \operatorname{Guards}\left(\mathrm{x}_{2} \cdot \mathrm{n}_{2}\right), & \text { if } \mathrm{m}=\delta\left(\mathrm{p}, \mathrm{x}_{1} \cdot \mathrm{n}_{1}, \mathrm{x}_{2} \cdot \mathrm{n}_{2}\right) \\
\emptyset, & \text { otherwise }\end{cases} \\
& \operatorname{Guards}(\mathrm{x} . \mathrm{n})=\{\mathrm{m} \in \operatorname{Guards}(\mathrm{n}) \mid \mathrm{x} \notin f v(\mathrm{~m})\} \\
& \text { Paths }(\mathrm{gs})=\left\{\rho \mid \rho \in\{1,2\}^{\mathrm{gs}}\right\} \quad v(\mathrm{ps})=\left\{\mathrm{p}_{\mathrm{x}_{2}}^{\mathrm{x}_{1}} \mid \mathrm{p} \in \mathrm{ps} / \sim \text { and } \mathrm{x}_{1}, \mathrm{x}_{2} \text { fresh }\right\} \\
& \operatorname{Tail}_{\rho\left[\mathrm{p}_{x_{2}}^{\left.x_{1} \mapsto i\right]}\right.}\left(\delta\left(\mathrm{p}^{\prime}, \mathrm{x}_{1}^{\prime} \cdot \mathrm{n}_{1}^{\prime}, \mathrm{x}_{2}^{\prime} \cdot \mathrm{n}_{2}^{\prime}\right)\right)=\operatorname{Tail}_{\rho\left[\mathrm{p}_{x_{2}} \mathrm{x}_{1} \mapsto i\right]}\left(\mathrm{n}_{\mathrm{i}}\left[\mathrm{x}_{\mathrm{i}}^{\prime}:=\mathrm{x}_{\mathrm{i}}\right]\right) \text {, if } \mathrm{p} \sim \mathrm{p}^{\prime} \\
& \operatorname{Tail}_{\rho}(\mathrm{m})=\mathrm{m}
\end{aligned}
$$


The function Guards(m) gives the set of independent guards at the top-level of $m$. The definition of Guards is the same as that used by Balat et al. If $\mathrm{ps}=\operatorname{Guards}(\mathrm{m})$, then Paths $(\mathrm{v}(\mathrm{ps}))$ represents the set of possible paths through $m$ dictated by $p s$. Given a path $\rho$ through a term $m$, the subterm of $m$ at the end of that path, the tail of $\rho$, is given by $\operatorname{Tail}_{\rho}(\mathrm{m})$. We write $\mathrm{ps} / \sim$ for the quotient set of $p s$ by $\sim$.

\section{Proposition 4.}

$$
\begin{aligned}
& \mathrm{m}_{1} \sim \mathrm{m}_{2} \Longleftrightarrow \\
& \quad \forall \rho \in \operatorname{Paths}\left(v\left(\operatorname{Guards}\left(\mathrm{m}_{1}\right) \cup \operatorname{Guards}\left(\mathrm{m}_{2}\right)\right)\right) . \operatorname{Tail}_{\rho}\left(\mathrm{m}_{1}\right) \sim \operatorname{Tail}_{\rho}\left(\mathrm{m}_{2}\right)
\end{aligned}
$$

To decide whether $m_{1} \sim m_{2}$ : if one of $m_{1}, m_{2}$ is a case, then use Prop. 4 otherwise compare the top-level constructors and if equal recurse on the immediate subterms of $m_{1}, m_{2}$. Having defined the decidable equivalence $\sim$, we now present the rewrite rules. The $\beta$ - and $\eta$-rules are standard.

$\beta$-rules

$$
\begin{aligned}
& (\rightarrow . \beta) \quad \lambda x \cdot m n \longrightarrow m[x:=n] \\
& \left(\times . \beta_{1}\right) \quad \pi_{1}\left(\left\langle\mathrm{~m}_{1}, \mathrm{~m}_{2}\right\rangle\right) \longrightarrow \mathrm{m}_{1} \\
& \left(\times . \beta_{2}\right) \quad \pi_{2}\left(\left\langle m_{1}, m_{2}\right\rangle\right) \longrightarrow m_{2} \\
& \left(+. \beta_{1}\right) \quad \delta\left(\iota_{1}(m), x_{1} \cdot n_{1}, x_{2} \cdot n_{2}\right) \longrightarrow n_{1}\left[x_{1}:=m\right] \\
& \left(+. \beta_{2}\right) \quad \delta\left(\mathfrak{l}_{2}(\mathrm{~m}), \mathrm{x}_{1} \cdot \mathrm{n}_{1}, \mathrm{x}_{2} \cdot \mathrm{n}_{2}\right) \longrightarrow \mathrm{n}_{2}\left[\mathrm{x}_{2}:=\mathrm{m}\right]
\end{aligned}
$$

$\eta$-rules The $\eta$-rules are type-directed expansions.

$$
\begin{array}{ll}
(\rightarrow . \eta) & m^{A \rightarrow B} \longrightarrow \lambda x \cdot m x, \quad \text { if } x \notin f v(m) \\
(\times . \eta) & m^{A \times B} \longrightarrow\left\langle\pi_{1}(m), \pi_{2}(m)\right\rangle \\
(+. \eta) & m^{A+B} \longrightarrow \delta\left(m, x_{1} \cdot l_{1}\left(x_{1}\right), x_{2} \cdot l_{2}\left(x_{2}\right)\right)
\end{array}
$$

The annotation $\mathrm{m}^{\mathrm{A}}$ means $\mathrm{m}$ has type $\mathrm{A}$. The $\eta$-rules are applicable only if expansion does not create a new redex. More precisely, only variables, applications and projections (pure neutral terms) can be $\eta$-expanded, and only in a non-elimination frame.

In order to instantiate the move-case axiom as a rewrite rule we read it from left to right. This corresponds to hoisting a case over a frame. Of course, we do not generally need to allow hoisting over continuation frames, as this is captured by $\sim$. However, for confluence it is necessary to allow hoisting over several continuation frames followed by a non-continuation frame. 
Frames and contexts

(Hoisting frames)

(Discriminator contexts)

(Hoisting contexts)

$$
\begin{aligned}
\mathrm{H}[]] & :=\mathrm{F}_{1}[]\left|\mathrm{F}_{2}[]\right| \mathrm{F}_{3}[] \\
\mathrm{D}[]] & :=[] \mid \delta\left(p, x_{1} . \mathrm{D}[], x_{2} \cdot \mathrm{n}_{2}\right) \\
& \mid \delta\left(p, x_{1} \cdot \mathrm{n}_{1}, x_{2} . \mathrm{D}[]\right) \\
\mathrm{HD}[] & ::=\mathrm{H}[\mathrm{D}[]]
\end{aligned}
$$

$\gamma$-rules We refer to reduction rules that arise from the move-case-, repeated-guardand redundant-guard-axioms as $\gamma$-rules.

$$
\begin{aligned}
& \text { (hoist-case) } \\
& \quad \operatorname{HD}\left[\delta\left(p, x_{1} \cdot n_{1}, x_{2} \cdot n_{2}\right)\right] \longrightarrow \delta\left(p, x_{1} \cdot \operatorname{HD}\left[n_{1}\right], x_{2} \cdot \operatorname{HD}\left[n_{2}\right]\right), \\
& \quad x_{1}, x_{2} \notin f v(H D) \text { and } b v(H D) \cap f v(p)=\emptyset
\end{aligned}
$$

The hoist-case-rule is obtained from the move-case axiom. It is a generalisation of the usual commuting conversions. Note that continuation frames are not hoisting frames, as naïvely hoisting over continuation frames would lead to non-termination. However, the equivalence $\sim$ includes the possibility of moving cases over continuation frames, and the hoist-case-rule does allow a case to be hoisted over a hoisting frame from inside a discrimination context.

The discrimination context is necessary in the hoist-case-rule because it is only sound to hoist a case over a lambda abstraction if the lambda-bound variable does not occur free in the guard. If hoisting from within a discrimination context is disallowed, then some cases become blocked from being hoisted outside the lambda by outer cases that depend on the bound variable. For instance, suppose D is restricted to be the empty context [ ], then the terms

$$
\lambda x . \delta\left(x, x_{1} . \delta\left(z, y_{1} \cdot y_{1}, y_{2} \cdot y_{2}\right), x_{2} \cdot x_{2}\right)
$$

and

$$
\delta\left(z, y_{1} \cdot \lambda x \cdot \delta\left(x, x_{1} \cdot y_{1}, x_{2} \cdot x_{2}\right), y_{2} \cdot \lambda x \cdot \delta\left(x, x_{1} \cdot y_{2}, x_{2} \cdot x_{2}\right)\right)
$$

become distinct normal forms, despite the fact that these terms are identified in the equational theory.

Remark For confluence it is not necessary to have a discrimination context in the hoist-case $1_{1}$ - and hoist-case 2 -rules, but here we gave the single general hoist-case-rule for the sake of uniformity.

Definition 5 (Reduction relations).

$\longrightarrow \beta=$ the compatible closure of the $\beta$-rules

$\longrightarrow \eta=$ the restricted compatible closure of the $\eta$-rules

$\longrightarrow \gamma=$ the compatible closure of the $\gamma$-rules

$\longrightarrow \gamma_{\mathrm{E}}=$ the compatible closure of hoist-case with $\mathrm{HD}$ restricted to $\mathrm{F}_{1}$

(i.e. the standard commuting conversion reduction relation)

$\longrightarrow \gamma^{\prime}=\longrightarrow \gamma \backslash \gamma_{\mathrm{E}}$

$\longrightarrow_{\mathrm{c}}=\longrightarrow_{\beta} \cup \longrightarrow_{\eta} \cup{ }_{\gamma_{\mathrm{E}}}$

$\longrightarrow \quad=\longrightarrow \beta \cup \longrightarrow \eta \cup \longrightarrow \gamma$ 


\section{Strong normalisation for $\beta \eta \gamma_{E}$-reduction}

Strong normalisation is standard for $\beta \eta \gamma_{\mathrm{E}}$-reduction 511 . In this section we present an adaptation of the strong normalisation proof given in the author's thesis [8, Chapter 3]. Our use of frame stacks alleviates difficulties with $\gamma_{E^{-}}$ reduction, and leads to a significantly simpler proof than Prawitz's original one [11.

Definition 6. A term $\mathrm{m}$ is strongly normalising with respect to a reduction relation $\mathrm{R}$, or $\mathrm{R}-\mathrm{SN}$, if all $\mathrm{R}$-reduction sequences starting from $\mathrm{m}$ are finite. $A$ reduction relation $\mathrm{R}$ is strongly normalising, or $\mathrm{SN}$, if all terms $\mathrm{m}$ are $\mathrm{R}-S N$. If $\mathrm{m}$ is $\mathrm{R}-S N$, then we write $\max _{\mathrm{R}}(\mathrm{m})$ for the maximum length of a reduction sequence starting from $\mathrm{m}$.

Definition 7 (frame stacks).

$$
\begin{aligned}
& \begin{array}{l}
\text { (elimination frames) } \\
\text { (frame stacks) } \\
\text { (stack length) }
\end{array} \mathrm{E}::=\mathrm{F}_{1} \\
& \mathrm{~S}::=I d \mid \mathrm{S} \circ \mathrm{E} \\
&|I d|=0 \\
&(\text { plugging })|\mathrm{S} \circ \mathrm{E}|=|\mathrm{S}|+1 \\
& I d[\mathrm{~m}]=\mathrm{m} \\
&(\mathrm{S} \circ \mathrm{E})[\mathrm{m}]=\mathrm{S}[(\mathrm{E}[\mathrm{m}])]
\end{aligned}
$$

Following Girard et al [6] we assume variables are annotated with types (it is straightforward, albeit somewhat tedious, to adapt the proof to use local typing contexts instead). We write $A \multimap B$ for the type of frame stack $S$, if $S[m]$ : B for all terms $m: A$.

\section{Definition 8 (frame stack reduction).}

$$
\mathrm{S} \longrightarrow_{\mathrm{c}} \mathrm{S}^{\prime} \stackrel{\text { def }}{\Longleftrightarrow} \forall \mathrm{m} . \mathrm{S}[\mathrm{m}] \longrightarrow_{\mathrm{c}} \mathrm{S}^{\prime}[\mathrm{m}]
$$

A frame stack $S$ is c-strongly normalising if all c-reduction sequences starting from $S$ are finite.

\section{Lemma 9.}

1. $\mathrm{S} \longrightarrow_{\mathrm{c}} \mathrm{S}^{\prime}$ iff $\mathrm{S} \neq I d$ and $\mathrm{S}[\mathrm{x}] \longrightarrow_{\mathrm{c}} \mathrm{S}^{\prime}[\mathrm{x}]$.

2. If $\mathrm{S} \longrightarrow{ }_{\mathrm{c}} \mathrm{S}^{\prime}$, for frame stacks $\mathrm{S}, \mathrm{S}^{\prime}$, then $\left|\mathrm{S}^{\prime}\right| \leqslant|\mathrm{S}|$.

3. If there exists $\mathrm{m}$ such that $\mathrm{S}[\mathrm{m}]$ is $\mathrm{c}-S N$, then $\mathrm{S}[\mathrm{x}]$ is $\mathrm{c}-S N$.

Proof. Induction on the structure of S.

\section{Definition 10 (reducibility).}

- Id is reducible. 
$-\mathrm{S} \circ[] \mathrm{n}:(\mathrm{A} \rightarrow \mathrm{B}) \multimap \mathrm{C}$ is reducible if $\mathrm{S}$ and $\mathrm{n}$ are reducible.

$-\mathrm{S} \circ \pi_{i}([]):(\mathrm{A} \times \mathrm{B}) \multimap \mathrm{C}$ is reducible if $\mathrm{S}$ is reducible.

$-\mathrm{S}:(\mathrm{A}+\mathrm{B}) \multimap \mathrm{C}$ is reducible if $\mathrm{S}\left[\mathrm{\iota}_{1}(\mathrm{~m})\right]$ is $\mathrm{c}-S N$ for all reducible $\mathrm{m}: \mathrm{A}$, and $\mathrm{S}\left[\mathrm{L}_{2}(\mathrm{n})\right]$ is $\mathrm{c}-S N$ for all reducible $\mathrm{n}: \mathrm{B}$.

$-\mathrm{m}: \mathrm{A}$ is reducible if $\mathrm{S}[\mathrm{m}]$ is $\mathrm{c}-S N$ for all reducible $\mathrm{S}: \mathrm{A} \multimap \mathrm{C}$.

Lemma 11. If $\mathrm{m}: \mathrm{A}$ is reducible then $\mathrm{m}$ is $\mathrm{c}-S N$.

Proof. Follows immediately from reducibility of $I d$ and the definition of reducibility on terms.

Lemma 12. $x: A$ is reducible.

Proof. By induction on A using Lemma 9 and Lemma 11.

Corollary 13. If $\mathrm{S}: \mathrm{A} \multimap \mathrm{C}$ is reducible then $\mathrm{S}$ is $\mathrm{c}-S N$.

Each type constructor has an associated $\beta$-rule. Each $\beta$-rule gives rise to an SN-closure property.

Lemma 14 (SN-closure).

$\rightarrow$ If $\mathrm{S}[\mathrm{m}[\mathrm{x}:=\mathrm{n}]]$ and $\mathrm{n}$ are $\mathrm{c}-S N$ then $\mathrm{S}[(\lambda \mathrm{x} . \mathrm{m}) \mathrm{n}]$ is $\mathrm{c}-S N$.

$\times .1$ If $\mathrm{S}[\mathrm{m}]$ and $\mathrm{n}$ are $\mathrm{c}-S N$ then $\mathrm{S}\left[\pi_{1}(\langle\mathrm{~m}, \mathrm{n}\rangle)\right]$ is $\mathrm{c}-S N$.

$\times .2$ If $\mathrm{S}[\mathrm{n}]$ and $\mathrm{m}$ are $\mathrm{c}-S N$ then $\mathrm{S}\left[\pi_{2}(\langle\mathrm{~m}, \mathrm{n}\rangle)\right]$ is $\mathrm{c}-S N$.

+.1 If $\mathrm{S}\left[\mathrm{n}_{1}\left[\mathrm{x}_{1}:=\mathrm{m}\right]\right], \mathrm{S}\left[\mathrm{n}_{2}\right]$ and $\mathrm{m}$ are $\mathrm{c}-S N$ then

$\mathrm{S}\left[\delta\left(\mathrm{l}_{1}(\mathrm{~m}), \mathrm{x}_{1} \cdot \mathrm{n}_{1}, \mathrm{x}_{2} . \mathrm{n}_{2}\right)\right]$ is $\mathrm{c}-S N$.

+.2 If $\mathrm{S}\left[\mathrm{n}_{2}\left[\mathrm{x}_{2}:=\mathrm{m}\right]\right], \mathrm{S}\left[\mathrm{n}_{1}\right]$ and $\mathrm{m}$ are $\mathrm{c}-S N$ then

$\mathrm{S}\left[\delta\left(\mathrm{t}_{2}(\mathrm{~m}), \mathrm{x}_{1} \cdot \mathrm{n}_{1}, \mathrm{x}_{2} \cdot \mathrm{n}_{2}\right)\right]$ is $\mathrm{c}-S N$.

Proof.

$\rightarrow, \times .1, \times .2$ : By induction on $\max _{\mathrm{c}}(\mathrm{S})+\max _{\mathrm{c}}(\mathrm{m})+\max _{\mathrm{c}}(\mathrm{n})$.

+.1: By induction on $|\mathrm{S}|+\max _{\mathrm{c}}\left(\mathrm{S}\left[\mathrm{n}_{1}\left[\mathrm{x}_{1}:=\mathrm{m}\right]\right]\right)+\max \left(\mathrm{S}\left[\mathrm{n}_{2}\right]\right)+\max _{\mathrm{c}}(\mathrm{m})$.

+.2 : By induction on $|\mathrm{S}|+\max _{\mathfrak{c}}\left(\mathrm{S}\left[\mathrm{n}_{2}\left[\mathrm{x}_{2}:=\mathrm{m}\right]\right]\right)+\max _{\mathrm{c}}\left(\mathrm{S}\left[\mathrm{n}_{1}\right]\right)+\max _{\mathfrak{c}}(\mathrm{m})$.

Now we obtain reducibility-closure properties for each type constructor.

\section{Lemma 15 (reducibility-closure).}

$\rightarrow$ If $\mathrm{m}[\mathrm{x}:=\mathrm{n}]$ is reducible for all reducible $\mathrm{n}$, then $\lambda \mathrm{x} . \mathrm{m}$ is reducible.

$\times$ If $\mathrm{m}, \mathrm{n}$ are reducible, then $\langle\mathrm{m}, \mathrm{n}\rangle$ is reducible.

+ If $\mathrm{m}$ is reducible, $\mathrm{n}_{1}\left[\mathrm{x}_{1}:=\mathrm{l}\right]$ is reducible for all reducible $\mathrm{l}$, and $\mathrm{n}_{2}\left[\mathrm{x}_{2}:=\mathrm{p}\right]$ is reducible for all reducible $\mathrm{p}$, then $\delta\left(\mathrm{m}, \mathrm{x}_{1} \cdot \mathrm{n}_{1}, \mathrm{x}_{2} \cdot \mathrm{n}_{2}\right)$ is reducible.

Proof. Each property follows from the corresponding part of Lemma 14 using Lemma 11 and Corollary 13.

Theorem 16. Let $\mathrm{m}$ be any term. Suppose $\mathrm{x}_{1}: A_{1}, \ldots, \mathrm{x}_{\mathrm{k}}: \mathrm{A}_{\mathrm{k}}$ includes all the free variables of $\mathrm{m}$. If $\mathrm{p}_{1}: \mathrm{A}_{1}, \ldots, \mathrm{p}_{\mathrm{k}}: A_{\mathrm{n}}$ are reducible then $\mathrm{m}\left[\mathrm{x}_{1}:=\mathrm{p}_{1}, \ldots, \mathrm{x}_{\mathrm{k}}:=\right.$ $\left.p_{\mathrm{k}}\right]$ is reducible.

Proof. By induction on the structure of terms using Lemma 15.

Theorem 17 (strong normalisation). All terms are c-SN.

Proof. Let $m$ be a term with free variables $x_{1}, \ldots, x_{k}$. By Lemma $12, x_{1}, \ldots, x_{k}$ are reducible. Hence, by Thm. 16, $m$ is c-SN. 


\section{$5 \quad$ Weak normalisation and confluence}

It is straightforward to prove that $\gamma$-reduction is strongly normalising.

Lemma 18. If $\mathrm{D}[\mathrm{x}], \mathrm{m}$ are $\gamma-S N$, then $\mathrm{D}[\mathrm{m}]$ is $\gamma-S N$.

Proof. By induction on $\max _{\gamma}(\mathrm{D}[\mathrm{x}])+\max _{\gamma}(\mathrm{m})$.

Lemma 19. If $\mathrm{p}, \mathrm{n}_{1}, \mathrm{n}_{2}$ are $\gamma-S N$, then $\delta\left(\mathrm{p}, \mathrm{x}_{1} \cdot \mathrm{n}_{1}, \mathrm{x}_{2} \cdot \mathrm{n}_{2}\right)$ is $\gamma-S N$.

Proof. By induction on $\left\langle\max _{\gamma}(\mathrm{p})\right.$, size $\left.(\mathrm{p}), \max _{\gamma}\left(\mathrm{n}_{1}\right)+\max _{\gamma}\left(\mathrm{n}_{2}\right)\right\rangle$. The only interesting case is

$$
\begin{aligned}
& \delta\left(D\left[\delta\left(p, x_{1} \cdot p_{1}, x_{2} \cdot p_{2}\right)\right], y_{1} \cdot n_{1}, y_{2} \cdot n_{2}\right) \\
& \quad \longrightarrow \gamma \delta\left(p, x_{1} \cdot D\left[\delta\left(p_{1}, y_{1} \cdot n_{1}, y_{2} \cdot n_{2}\right)\right], x_{2} . D\left[\delta\left(p_{2}, y_{1} \cdot n_{1}, y_{2} \cdot n_{2}\right)\right]\right)
\end{aligned}
$$

By the induction hypothesis, $\delta\left(p_{1}, y_{1} \cdot n_{1}, y_{2} \cdot n_{2}\right)$ and $\delta\left(p_{2}, y_{1} \cdot n_{1}, y_{2} \cdot n_{2}\right)$ are both $\gamma$-SN. Then by Lemma 18 and the induction hypothesis

$$
\delta\left(p, x_{1} \cdot D\left[\delta\left(p_{1}, y_{1} \cdot n_{1}, y_{2} \cdot n_{2}\right)\right], x_{2} \cdot D\left[\delta\left(p_{2}, y_{1} \cdot n_{1}, y_{2} \cdot n_{2}\right)\right]\right)
$$

is $\gamma$-SN.

\section{Lemma 20.}

1. If $\mathrm{m}, \mathrm{n}$ are $\gamma-S N$ then $\mathrm{m} \mathrm{n}$ is $\gamma-S N$.

2. If $\mathrm{m}, \mathrm{n}$ are $\gamma-S N$ then $\langle\mathrm{m}, \mathrm{n}\rangle$ is $\gamma-S N$.

3. If $\mathrm{m}$ is $\gamma-S N$ then $\lambda x . \mathrm{m}$ is $\gamma-S N$.

4. If $\mathrm{m}$ is $\gamma-S N$ then $\pi_{\mathrm{i}}(\mathrm{m})$ is $\gamma-S N$.

5. If $\mathrm{m}$ is $\gamma-S N$ then $\mathrm{\iota}_{\mathrm{i}}(\mathrm{m})$ is $\gamma-S N$.

Proof.

1-2 By induction on $\left\langle\max _{\gamma}(\mathrm{m})+\max _{\gamma}(\mathrm{n})\right.$, size $\left.\left.(\mathrm{m})+\operatorname{size}(\mathrm{n})\right)\right\rangle$.

3-5 By induction on $\left\langle\max _{\gamma}(\mathrm{m})\right.$, size $\left.(\mathrm{m})\right\rangle$.

Theorem 21. $\gamma$ is strongly normalising.

We now obtain weak normalisation for $\beta \eta \gamma$-reduction. The key observation is that $\gamma$-reduction following $\beta \eta \gamma_{\mathrm{E}}$-normalisation cannot introduce new $\beta \eta$ redexes.

Lemma 22. If $\mathrm{m}$ is in $\beta \eta \gamma_{\mathrm{E}}$-normal form and $\mathrm{m} \longrightarrow{ }_{\gamma}^{*} \mathrm{~m}^{\prime}$, then $\mathrm{m}^{\prime}$ is in $\beta \eta$ normal form.

Lemma 22 is easily proved by a straightforward syntactic analysis of the structure of the term $\mathrm{m}^{\prime}$. The details are omitted due to lack of space.

Theorem 23. $\longrightarrow$ is weakly normalising. 
Proof. To normalise a term of $m$, first reduce to a $\beta \eta \gamma_{\mathrm{E}}$-normal form $\mathrm{m}^{\prime}$, then reduce $\mathrm{m}^{\prime}$ to $\gamma$-normal form $\mathrm{m}^{\prime \prime}$. By Lemma 22 , $\mathrm{m}^{\prime \prime}$ must be a $\beta \eta \gamma$-normal form.

We could obtain confluence by appealing to correctness of normalisation by evaluation for sums [1]. Instead, we give a direct proof of confluence modulo using the strong normalisation results for $\longrightarrow_{\mathrm{c}}$ and $\longrightarrow_{\gamma}$.

We write $R^{*}$ for the transitive reflexive closure of the relation $R$.

Definition 24. A reduction relation $\mathrm{R}$ is:

- confluent modulo $\sim$ iff for all $\mathrm{m}, \mathrm{n}, \mathrm{m}^{\prime}, \mathrm{n}^{\prime}$ with $\mathrm{m} \sim \mathrm{n}, \mathrm{m} \longrightarrow_{\mathrm{R}}^{*} \mathrm{~m}^{\prime}$ and $\mathrm{n} \longrightarrow{ }_{\mathrm{R}}^{*} \mathrm{n}^{\prime}$, there exist $\mathrm{m}^{\prime \prime}, \mathrm{n}^{\prime \prime}$ with $\mathrm{m}^{\prime} \longrightarrow_{\mathrm{R}}^{*} \mathrm{~m}^{\prime \prime}, \mathrm{n}^{\prime} \longrightarrow_{\mathrm{R}}^{*} \mathrm{n}^{\prime \prime}$ and $\mathrm{m}^{\prime \prime} \sim \mathrm{n}^{\prime \prime}$.

- weakly confluent modulo $\sim$ iff for all $\mathrm{m}, \mathrm{n}, \mathrm{p}$ with $\mathrm{m} \longrightarrow_{\mathrm{R}} \mathrm{n}$ and $\mathrm{m} \longrightarrow_{\mathrm{R}} \mathrm{p}$, there exist $\mathrm{n}^{\prime}, \mathrm{p}^{\prime}$ with $\mathrm{n} \longrightarrow{ }_{\mathrm{R}}^{*} \mathrm{n}^{\prime}, \mathrm{p} \longrightarrow{ }_{\mathrm{R}}^{*} \mathrm{p}^{\prime}$ and $\mathrm{n}^{\prime} \sim \mathrm{p}^{\prime}$.

- weakly coherent modulo $\sim$ iff for all $\mathrm{m}, \mathrm{n}, \mathrm{n}^{\prime}$ with $\mathrm{m} \sim \mathrm{n}$ and $\mathrm{n} \longrightarrow_{\mathrm{R}} \mathrm{n}^{\prime}$, there exist $\mathrm{m}^{\prime}, \mathrm{n}^{\prime \prime}$ with $\mathrm{m} \longrightarrow{ }_{\mathrm{R}}^{*} \mathrm{~m}^{\prime}, \mathrm{n}^{\prime} \longrightarrow{ }_{\mathrm{R}}^{*} \mathrm{n}^{\prime \prime}$ and $\mathrm{m}^{\prime} \sim \mathrm{n}^{\prime \prime}$.

Confluence, weak confluence, and weak coherence, all modulo
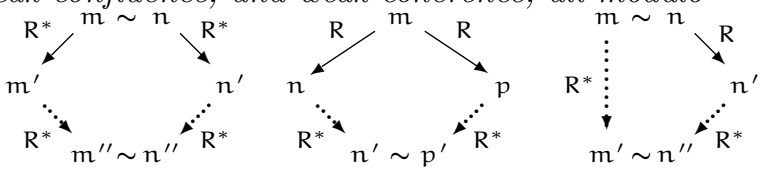

Theorem 25 (Huet's Theorem [7]). If the reduction relation $\mathrm{R}$ is strongly normalising, weakly confluent modulo $\sim$ and weakly coherent modulo $\sim$, then $\mathrm{R}$ is also confluent modulo $\sim$.

Proposition 26.

$\longrightarrow_{\beta}, \longrightarrow_{\eta}, \longrightarrow_{\mathrm{c}}, \longrightarrow_{\gamma}, \longrightarrow$ are all weakly confluent modulo $\sim$.

Proposition 27.

$\longrightarrow_{\beta}, \longrightarrow_{\mathfrak{\eta}}, \longrightarrow_{\mathfrak{c}}, \longrightarrow_{\gamma}, \longrightarrow$ are all weakly coherent modulo $\sim$.

Proposition 28.

$\longrightarrow_{\beta}, \longrightarrow_{\eta}, \longrightarrow_{\mathrm{c}}, \longrightarrow_{\gamma}$ are all confluent modulo $\sim$.

Proof. By Huet's Theorem using Prop. 26, Prop. 27, Thm. 17 and Thm. 21

We now show confluence of $\longrightarrow$ modulo $\sim$ using some intermediate Lemmas. The only non-trivial interaction is between $\beta$ - and $\gamma^{\prime}$-reduction. Following Barendregt [3, Chapter 11] we allow $\beta$-redexes to be marked. A redex is marked by overlining it. Notice that $\gamma^{\prime}$-reduction can hide $\beta$-redexes inside a $\gamma_{\mathrm{E}}$-redex. In such cases, we allow the $\gamma_{\mathrm{E}}$-redex to be marked. For instance

$$
\overline{\left(\lambda x . \delta\left(p, x_{1} \cdot n_{1}, x_{2} \cdot n_{2}\right)\right) m} \longrightarrow \gamma^{\prime} \overline{\delta\left(p, x_{1} . \lambda x . n_{1}, x_{2} . \lambda x . n_{2}\right) m}
$$




\section{Definition 29.}

$$
\begin{aligned}
\varphi(\overline{(\lambda x \cdot m) n}) & =\varphi(m)[x:=\varphi(n)] \\
\varphi\left(\overline{\pi_{i}(\langle m, n\rangle)}\right) & =\varphi(m) \\
\varphi\left(\overline{\delta\left(\mathfrak{l i}_{i}(m), x_{1} \cdot n_{1}, x_{2} \cdot n_{2}\right)}\right) & =\varphi\left(n_{i}\right)\left[x_{i}:=\varphi(m)\right] \\
\varphi\left(\overline{E\left[\delta\left(p, x_{1} \cdot n_{1}, x_{2} \cdot n_{2}\right)\right]}\right) & =\delta\left(\varphi(p), x_{1} \cdot \varphi\left(\overline{E\left[n_{1}\right]}\right), x_{2} \cdot \varphi\left(\overline{E\left[n_{2}\right]}\right)\right)
\end{aligned}
$$

$\varphi$ commutes with all the other syntax constructors.

The $\varphi$ function contracts all of the marked $\beta$-redexes in a term.

Lemma 30. $\varphi(m[x:=n])=\varphi(m)[x:=n]$

Proof. By induction on the structure of $\mathrm{m}$.

Lemma 31. If $\mathrm{m} \sim \mathrm{m}^{\prime}$ then $\varphi(\mathrm{m}) \sim \varphi\left(\mathrm{m}^{\prime}\right)$.

Proof. By induction on the derivation of $m \sim \mathrm{m}^{\prime}$.

Lemma 32. (a)

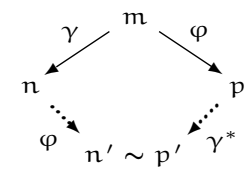

(b)

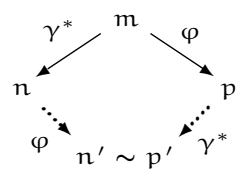

Proof.

(a) By induction on the derivation of $\gamma$ using Lemma 30 and Lemma 31 .

(b)

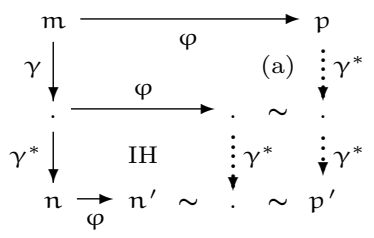

Lemma 33.

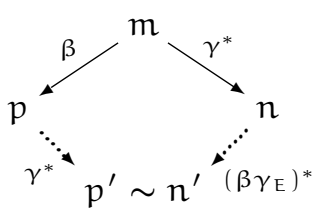

Proof. Let $\bar{m}$ be $m$ with the $\beta$-redex marked, and $\|$ be an operator that erases marked redexes, but otherwise leaves a term unchanged.

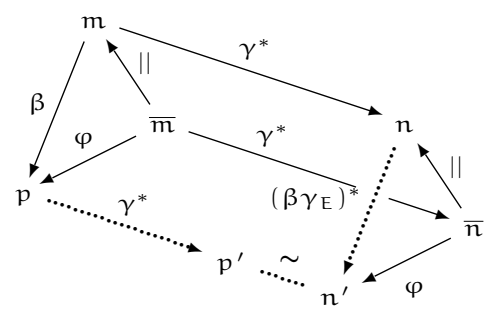

The front triangle is proved by induction on the structure of $\bar{n}$. The bottom rectangle is proved by Lemma 32 
Proposition 34.

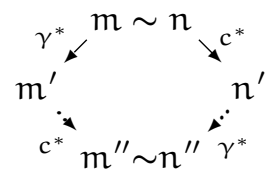

Proof. Using Lemma 33 .

Theorem 35. $\longrightarrow$ is confluent modulo $\sim$.

Proof. By a diagram chase using Prop. 28 and Prop. 34

Theorem 36. $\lambda^{\rightarrow \times+}$ is decidable.

Proof. By Thm. 35, and Thm. 23, every $\lambda^{-\times+}$-term has a unique normal form obtained by reducing to $\beta \eta \gamma_{\mathrm{E}}$-normal form and then to $\gamma$-normal form. To decide whether terms $m, n$ are equal simply reduce them to normal forms $m^{\prime}, n^{\prime}$ and then compute whether $\mathrm{m}^{\prime} \sim \mathrm{n}^{\prime}$.

\section{Variations}

Unblocking cases It would be nice if it was possible to remove discrimination contexts from the move-case ${ }_{3}$-rule, and so allow all the rewrite rules to be local. One way of doing so is to mark a case as blocked when it is adjacent to a lambda abstraction on whose bound variable the guard depends. Then unblocked cases can be lifted over blocked cases. The resulting calculus is somewhat fiddly, though, as blocked cases can subsequently become unblocked by $\beta$-reductions inside the guard. We omit the details, and instead consider some more wellbehaved alternatives.

Parallel cases Altenkirch et al [1] use parallel cases in order to define normalisation by evaluation for sums. We write

$$
\Delta\left(\left[\left(x_{0}, p_{0}\right), \ldots,\left(x_{l-1}, p_{l-1}\right)\right],\left[e_{0}, \ldots, e_{2^{l}-1}\right]\right)
$$

for the parallel case over the guards $p_{0}, \ldots, p_{l-1}$ with binders $x_{0}, \ldots, x_{l-1}$ and tails $e_{0}, \ldots, e_{2^{l}-1}$.

An easy way to comprehend the syntax is via the erasure ser from parallel cases to a tree of nested serial cases.

$$
\begin{aligned}
\operatorname{ser}\left(\Delta\left((x, p):: g s, e s_{1}+e s_{2}\right)\right) & =\delta\left(p, x \cdot \operatorname{ser}\left(\Delta\left(g s, e s_{1}\right)\right), x \cdot \operatorname{ser}\left(\Delta\left(g s, e s_{2}\right)\right)\right) \\
\operatorname{ser}(\Delta(\square,[e])) & =e
\end{aligned}
$$

The ser function commutes with all other syntax constructors. The operator :: appends an element to the front of a list. The operator ++ concatenates two lists of equal length. The translation par from a $\lambda^{-\times+}$-term to a term with parallel cases, simply converts each serial case into a parallel case with one guard.

$$
\operatorname{par}\left(\delta\left(\mathrm{p}, \mathrm{x} . \mathrm{n}_{1}, \mathbf{x} . \mathrm{n}_{2}\right)\right)=\Delta\left((\mathrm{x}, \operatorname{par}(\mathrm{p})),\left[\operatorname{par}\left(\mathrm{n}_{1}\right), \operatorname{par}\left(\mathrm{n}_{2}\right)\right]\right)
$$

The par function commutes with all other syntax constructors. 
Definition 37 . The relation $\approx$ is the least congruence such that for all permutations perm of the integers $1, \ldots, n$ :

$$
\Delta\left([,[e]) \approx e \quad \Delta\left(g s,\left[e_{0}, \ldots, e_{2^{l}-1}\right]\right) \approx \Delta\left(g^{\prime},\left[e_{0}^{\prime}, \ldots, e_{2^{l}-1}^{\prime}\right]\right)\right.
$$

where

$$
\begin{aligned}
& \mathrm{gs}=\left(\mathrm{x}_{0}, \mathrm{p}_{0}\right) \ldots\left(\mathrm{x}_{\mathrm{l-1}}, \mathrm{p}_{\mathrm{l-1}}\right) \quad \mathrm{e}_{\mathrm{i}}^{\prime}=\mathrm{e}_{\text {perm* } * \mathrm{i})} \\
& \mathrm{gs}^{\prime}=\left(\mathrm{x}_{0}^{\prime}, \mathrm{p}_{0}^{\prime}\right) \ldots\left(\mathrm{x}_{l_{-1}}^{\prime}, \mathrm{p}_{l_{-1}}^{\prime}\right) \text { with } \mathrm{x}_{\mathrm{i}}^{\prime}=\mathrm{x}_{\text {perm }(\mathrm{i})} \text { and } \mathrm{p}_{\mathrm{i}}^{\prime}=\mathrm{p}_{\text {perm }(\mathrm{i})} \\
& \operatorname{perm} *(\mathfrak{i})=\uparrow\left(\operatorname{perm}_{\mathcal{Q}}(\downarrow \mathfrak{i})\right) \quad \operatorname{perm}_{\mathcal{Q}}\left(\mathrm{b}_{1-1} \ldots \mathrm{b}_{0}\right)=\mathrm{b}_{\text {perm }(l-1)} \ldots \mathrm{b}_{\text {perm }(0)}
\end{aligned}
$$

$\downarrow, \uparrow$ convert natural numbers to and from binary

Given two terms $m_{1}$ and $m_{2}$, then $m_{1} \approx m_{2}$ iff $m_{2}$ can be obtained from $m_{1}$ by permuting guards (and adjusting binders and tails accordingly).

$\beta$ - and $\eta$-rules The $\beta$ - and $\eta$-rules are as in the serial rewriting theory, except for sums, where they are translated in the obvious way.

$$
\begin{aligned}
& \left(+. \beta_{1}\right) \quad \Delta\left(\left(x, \iota_{1}(m)\right):: \mathrm{gs}, e s_{1}++e s_{2}\right) \longrightarrow \Delta\left(\mathrm{gs}, e s_{1}[x:=\mathrm{m}]\right) \\
& \left(+. \beta_{2}\right) \quad \Delta\left(\left(x, \mathrm{l}_{2}(\mathrm{~m})\right):: \mathrm{gs}, e s_{1}+\mathrm{es}_{2}\right) \longrightarrow \Delta\left(\mathrm{gs}, e s_{2}[\mathrm{x}:=\mathrm{m}]\right) \\
& (+. \eta) \quad m^{A+B} \longrightarrow \Delta\left((x, m),\left[\iota_{1}(x), \mathfrak{l}_{2}(x)\right]\right)
\end{aligned}
$$

For sum types, $\beta$-rules are only needed for the first guard of a parallel elimination. Other guards can just be eliminated by first applying $\approx$. 
$\gamma$-rules

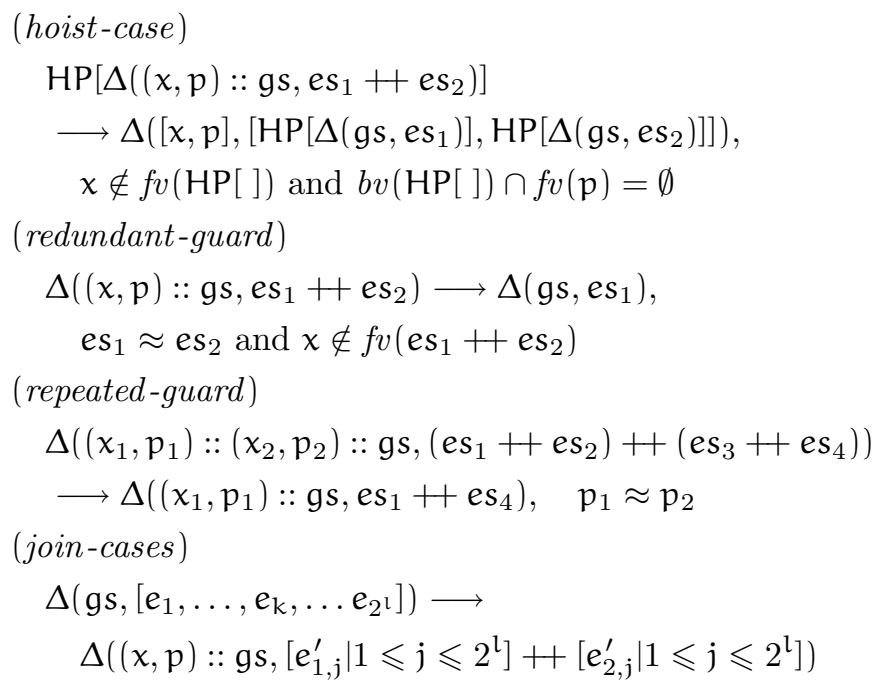

where

$$
\begin{aligned}
& e_{k}=\Delta\left((x, p):: g^{\prime}, e s_{1}++e s_{2}\right) \\
& \{\mathbf{x}\} \notin \operatorname{Binders}(\mathrm{gs}) \text { and }(\operatorname{Binders}(\mathrm{gs}) \cap f v(\mathbf{p}))=\emptyset \\
& e_{i, j}^{\prime}= \begin{cases}e_{j}, & \text { if } j \neq k \\
\Delta\left(\mathrm{gs}^{\prime}, e s_{i}\right), & \text { otherwise }\end{cases} \\
& \operatorname{Binders}\left(\Delta\left(\left[\left(x_{0}, p_{0}\right), \ldots,\left(x_{l-1}, p_{l-1}\right)\right],\left[e_{0}, \ldots, e_{2^{l}-1}\right]\right)\right)=\left\{x_{0}, \ldots, x_{l-1}\right\} \\
& \mathrm{HP}[]::=[] \mathrm{n}\left|\pi_{1}([])\right| \pi_{2}([]) \mid \Delta((x,[]):: \mathrm{gs}, \text { es }) \\
& |\lambda x .[]| m[]|\langle[], n\rangle|\langle m,[]\rangle\left|\mathfrak{l}_{1}([])\right| \mathfrak{l}_{2}([])
\end{aligned}
$$

The redundant-guard- and repeated-guard-rules are both obtained by reading the corresponding axioms from left to right. The move-case ${ }_{4}$ axiom is captured by the combination of: parallel cases, the relation $\approx$ and the join-cases-rule; which allows a guard of a tail to be merged with the guards of its parent parallel case, providing the guard is independent of the guards of the parent.

Definition 38. $\longrightarrow \mathrm{p}=$ the union of the compatible closure of the above $\beta$ - and $\gamma$-rules, and the restricted compatible closure of the above $\eta$-rules.

The proofs of Sections 4 and 5 are easily adapted to handle parallel cases.

Proposition 39. $\longrightarrow \mathrm{p} / \approx$ is weakly normalising and confluent.

Simulating parallel cases It is possible to simulate parallel cases using plain $\lambda^{\rightarrow+}{ }^{+}$-syntax. The key to avoiding non-termination is to define a congruence such that guards can only be duplicated if in normal form. 
Definition 40. The relation $\approx^{\prime}$ is the least congruence such that

$$
\begin{aligned}
& \delta\left(p_{1}, x_{1} \cdot \delta\left(p_{2}, y_{1} \cdot n_{1}, y_{2} \cdot n_{2}\right), x_{2} \cdot \delta\left(p_{2}, y_{1} \cdot n_{3}, y_{2} \cdot n_{4}\right)\right) \\
& \quad \approx^{\prime} \delta\left(p_{2}, y_{1} \cdot \delta\left(p_{1}, x_{1} \cdot n_{1}, x_{2} \cdot n_{3}\right), y_{2} \cdot \delta\left(p_{1}, x_{1} \cdot n_{2}, x_{2} \cdot n_{4}\right)\right), \\
& \quad x_{1}, x_{2}, y_{1}, y_{2} \notin f v\left(p_{1}\right) \cup f v\left(p_{2}\right) \\
& \delta\left(p_{1}, x_{1} \cdot \delta\left(p_{2}, y_{1} \cdot n_{1}, y_{2} \cdot n_{2}\right), x_{2} \cdot n_{3}\right) \\
& \quad \sigma^{\prime} \delta\left(p_{1}, x_{1} \cdot \delta\left(p_{2}, y_{1} \cdot n_{1}, y_{2} \cdot n_{2}\right), x_{2} \cdot \delta\left(p_{2}, y_{1} \cdot n_{3}, y_{2} \cdot n_{3}\right)\right), \\
& \quad x_{2} \notin f v\left(p_{2}\right) \text { and } y_{1}, y_{2} \notin f v\left(n_{3}\right) \\
& \delta\left(p_{1}, x_{1} \cdot n_{1}, x_{2} \cdot \delta\left(p_{2}, y_{1} \cdot n_{2}, y_{2} \cdot n_{3}\right)\right) \\
& \quad \approx^{\prime} \delta\left(p_{1}, x_{1} \cdot \delta\left(p_{2}, y_{1} \cdot n_{1}, y_{2} \cdot n_{1}\right), x_{2} \cdot \delta\left(p_{2}, y_{1} \cdot n_{2}, y_{2} \cdot n_{3}\right)\right) \\
& \quad x_{1} \notin f v\left(p_{2}\right) \text { and } y_{1}, y_{2} \notin f v\left(n_{1}\right)
\end{aligned}
$$

where in each case $p_{1}, p_{2}$ must be in normal form.

$\gamma$-rules

$$
\begin{aligned}
& \text { (hoist-case) } \\
& \mathrm{H}\left[\delta\left(p, x_{1} \cdot n_{1}, x_{2} \cdot n_{2}\right)\right] \longrightarrow \delta\left(p, x_{1} \cdot H\left[n_{1}\right], x_{2} \cdot H\left[n_{2}\right]\right), \\
& \mathrm{x}_{1}, \mathrm{x}_{2} \notin f v(\mathrm{H}) \text { and } b v(\mathrm{H}) \cap f v(\mathrm{p})=\emptyset \\
& \text { (duplicate-guard) } \\
& \delta\left(p, x_{1} . \delta\left(p, y_{1} \cdot m_{1}, y_{2} \cdot m_{2}\right), x_{2} . \delta\left(p, y_{1} \cdot n_{1}, y_{2} \cdot n_{2}\right)\right) \\
& \longrightarrow \delta\left(p, x_{1} \cdot m_{1}\left[y_{1}:=x_{1}\right], x_{2} \cdot n_{2}\left[y_{2}:=x_{2}\right]\right), \quad x_{1}, x_{2} \notin f v(p) \\
& \text { (redundant-guard) } \\
& \mathrm{C}\left[\delta\left(\mathrm{p}, \mathrm{x}_{1} \cdot \mathrm{n}, \mathrm{x}_{2} \cdot \mathrm{n}\right)\right] \longrightarrow \mathrm{C}[\mathrm{n}], \quad \mathrm{x}_{1}, \mathrm{x}_{2} \notin f v(\mathrm{n}) \text { and } \\
& \mathrm{C} \equiv[] \text {; or } \\
& \mathrm{C} \text { is a hoisting frame; or } \\
& \mathrm{C} \text { is a continuation frame with }(b v(\mathrm{C}) \cap f v(\mathrm{p})) \neq \emptyset
\end{aligned}
$$

The constraints on the context in which redundant-guard can be applied are necessary in order to prevent cycles with $\approx^{\prime}$.

Definition 41. $\longrightarrow_{p}=$ the union of $\longrightarrow_{\beta} \cup \longrightarrow_{\eta}$ and the compatible closure of the above $\gamma$-rules.

Proposition 42. $\longrightarrow \mathrm{p}_{/ \sim}$, is weakly normalising and confluent.

Conjecture 43. $\longrightarrow, \longrightarrow \mathrm{P} / \approx, \longrightarrow \mathrm{p} / \sim^{\prime}$ are all strongly normalising.

Intuitively, it seems that $\longrightarrow$ should be strongly normalising. Both c-reduction and $\gamma$-reduction are strongly normalising, and $\gamma^{\prime}$-reduction only interacts with c-reduction in such a way as to expose existing redexes, rather than actually creating new ones. If we could prove strong normalisation for $\longrightarrow$, then the confluence proof could be simplified. 


\section{Conclusion}

We have proposed a confluent extensional rewriting theory for simply-typed lambda-calculus extended with sums. The key contribution is confluence and decidability for a conventional rewriting theory. This contrasts with the two previous approaches to decidability. Ghani $[5$ uses intricate rewriting techniques, whereas Altenkirch et al [1] use normalisation by evaluation and category theory.

Acknowledgements Thanks to Philip Wadler and the anonymous reviewers for helpful feedback.

\section{References}

1. T. Altenkirch, P. Dybjer, M. Hofmann, and P. Scott. Normalization by evaluation for typed lambda calculus with coproducts. In 16th Annual IEEE Symposium on Logic in Computer Science, pages 303-310, Boston, Massachusetts, June 2001.

2. V. Balat, R. D. Cosmo, and M. Fiore. Extensional normalisation and type-directed partial evaluation for typed lambda calculus with sums. In 31st Symposium on Principles of Programming Languages (POPL 2004), pages 64-76. ACM Press, Jan. 2004.

3. H. P. Barendregt. The Lambda Calculus: Its Syntax and Semantics. Number 103 in Studies in Logics and the Foundations of Mathmatics. North Holland, 1984.

4. U. Berger, M. Eberl, and H. Schwichtenberg. Normalization by evaluation. In Prospects for Hardware Foundations (NADA), number 1546 in Lecture Notes in Computer Science, pages 117-137, 1998.

5. N. Ghani. Beta-eta equality for coproducts. In Proceedings of TLCA'95, number 902 in Lecture Notes in Computer Science, pages 171-185. Springer-Verlag, 1995.

6. J.-Y. Girard, Y. Lafont, and P. Taylor. Proofs and Types. Cambridge University Press, 1989.

7. G. P. Huet. Confluent reductions: Abstract properties and applications to term rewriting systems. J. ACM, 27(4):797-821, 1980.

8. S. Lindley. Normalisation by Evaluation in the Compilation of Typed Functional Programming Languages. PhD thesis, University of Edinburgh, 2005.

9. S. Lindley and I. Stark. Reducibility and TT-lifting for computation types. In P. Urzyczyn, editor, TLCA, volume 3461 of Lecture Notes in Computer Science, pages 262-277. Springer, 2005.

10. Y. Ohta and M. Hasegawa. A terminating and confluent linear lambda calculus. In $R T A$, pages $166-180,2006$.

11. D. Prawitz. Ideas and results in proof theory. In Proceedings of the 2nd Scandinavian Logic Symposium, number 63 in Studies in Logics and the Foundations of Mathmatics, pages 235-307. North Holland, 1971. 